\title{
Interview
}

\section{Ooyala - Accelerating the evolution of online video - An Interview with Sean Knapp of online video publishing}

\section{Sean Knapp}

is Ooyala's Co-founder and Chief Technology Officer, overseeing all engineering at Ooyala. Sean came from Google, where he developed and launched iGoogle. Sean built out the web search team, which was responsible for a billion dollar revenue increase. He holds a BS and an MS from Stanford University.

\section{ABSTRACT Ooyala CTO Sean Knapp discusses the evolution of online video publishing (from less than $\mathbf{5}$ years ago) and where it is headed today.}

Journal of Digital Asset Management (2009) 5, 264-273. doi:10.1057/dam.2009.23

Keywords: online video; interactive; monetization; CDN; white-label-video; Internet TV

MM: We're here with Ooyala. Could we start off with a personal introduction?

SN: Sure. My name is Sean Knapp. I'm co-founder and CTO of Ooyala.

Prior to Ooyala, I was at Google for several years. I led teams on web search, search, ads, interfaces - as well as site speed.

I was also one of the original engineers on iGoogle. I did the first two launches, there.

Prior to my time at Google, I was getting my undergraduate and graduate degrees in computer science at Stanford.

MM: How about giving us a little bit of a background of Ooyala?

SN: Myself along with two coworkers from Google founded the company in April of 2007, Bismarck and Belsasar Lepe.

The short history around it is - the name comes from a Southern Indian language - Telugu. 'Ooyala' means 'Cradle'. We thought that we were cradling innovation. And the dot-com URL was available. So it was a good fit.

When we originally left Google, we were building, essentially, a portal. Our primary focus was on interactive video, and on making video more engaging, and consequently, easier to monetize.

In the course of building the technology platform, we ended up solving a lot of what we consider to be fundamental problems with video consumption online - everything from content storage and transcoding to management, analytics, monetization and syndication.

We quickly found there was a huge demand for a company that could provide that suite of services that really was innovating in each one of those domains. Not just simply a reseller.

In our first 6 months, we transformed the company into a video enterprise and service company to provide the tools and functionality that you see today at Ooyala.

MM: One of the things that we've talked about is the idea of the emergence of a business ecosystem, serving media, entertainment and publishing, and the marketing operations.

In this ecosystem, we find companies such as Ooyala that have begun to emerge as a center of excellence in one particular area of the media supply chain.

Rather than just focusing on one aspect, such as transcoding or delivery, you kind of say, 'Okay. Let's really understand how we can innovate across a deep set of capabilities, as it relates to one thing. For example let's take online video'.

Perhaps you can provide a historical overview of online video, how it's evolved, and bring us up to date on the current state.

SN: Sure.

If we really look at the history here, until probably the last couple of years, there's not really been any easy way to solve the problem 
of having content and users, and needing to somehow connect the two.

It was a very difficult question or problem to solve independently - or simply by outsourcing 2 years ago. This really stemmed from the fact that there was no great application or plug-in model within the browsers, outside of Flash delivering video. There was no real services company, no real community that built up a lot of expertize to help - en masse - a lot of the content owners.

Until the proliferation of online video platform companies, you needed to grab your video and do some manual transcoding of each of those video assets. You'd most likely upload them manually to your content delivery network (CDN), and then you'd have a fairly basic Flash player that you'd point those video files at. Then you'd just download them from your CDN.

There really wasn't much complexity there. It was a pretty inefficient system, and it simply didn't scale well.

Talking to your point, we really came in as somebody who actually had the same needs as a lot of our customers do, today. We just simply began solving the most pressing problems, and did it as fast and efficiently as possible.

That very quickly enabled us to become a very successful and very beneficial enterprise play for a lot of our customers. A lot of our philosophy, I think, is that we're engineering 'Down the evolutionary path'. The very basic part of video delivery online is, 'I have this content. I have these users. I need to connect the two'.

Those are the basics. Storage, transcoding and delivery type model. A fairly basic contentmanagement system.

But once a content owner really hits that phase, very quickly you're looking to monetize your content. You're looking to analyze your content and actually see how much of that content is being consumed by your users. You're looking to produce better content - produce a better user-experience delivering and powering your content.

There's a natural evolution from there into some of the more exotic elements, around new monetization models. Interactive video. Better user-experience. That really follows a lot of our product suite.
MM: So you'd say that we're more or less in the third phase of online video?

SN: I think it actually depends on the customer. A lot of our content owners today will use our storage - our content management, our delivery and transcoding. Maybe they'll use a little bit of monetization. They'll have their integration with DoubleClick or Lightningcast or one of the other ad providers that we integrate. They'll use some of the analytics, but they won't necessarily be as advanced as Bebo, for example. They're using our computer vision and interactive video product offering to augment their content and make a more interactive video experience.

MM: So it goes to the idea expressed originally by the sci-fi author William Gibson - 'The future arrives unevenly distributed'.

SN: That's true. We have a very broad distribution across that spectrum for our content owners. Our real goal is to continue to push along that spectrum. So as they grow their businesses, we're always one step ahead on the product offering. And hopefully, we can help accelerate that growth for them, as well.

MM: I'd like to weave into the discussion of the history of online video, some of the distinctions or innovations around browsers and media players.

Traditionally, we've had two groups. We've had, 'Download the video', and 'Play it', which is Windows Media Player or QuickTime. They you had the streaming video players - originally, it was Real Networks and then Adobe Flash and then later Microsoft Silverlight.

Can you just bring us up to speed on how all of those things have shaken out today? SN: Sure. On a basic level, not every mainstream browser has native video support. Microsoft IE doesn't. Firefox doesn't. Safari doesn't. Mozilla is the only browser that's put an investment into building a browser-native codec for video.

The way that every browser is able to play back video is through plug-ins. That's a key component, as far as history goes, because that really dictates a lot of the direction that everybody has taken.

If you look at the most dominant plug-ins today across the browsers, Flash is clearly the winner. They're pretty much ubiquitous with an over 99 per cent browser penetration. Behind them is QuickTime, Real, Windows 
Media Player and SilverLight. Last I looked,

SilverLight was about 25 per cent penetration.

Because you need one of those plug-ins to really run a video-based application, you can see how people have really placed their bets and made their decisions around which plug-in environment they're going to build their video-based applications.

MM: Do you anticipate that - moving forward video applications will continue to be plug-ins for most Internet-connected devices? Or do you anticipate that at some point, they will be built into the operating system or the browser? SN: I see it getting bundled into the browser, eventually. I see Mozilla has taken that first step with the $100 \mathrm{~K}$ investments into research around a new codec that would be natively supported in the Mozilla browser.

But if you look at browser trends, even if that were to be successful and to be adopted by the vast majority of browsers, moving forward we're still looking at 2012 or 2013, before you're even close to a 90 per cent penetration, where that model works.

A lot of our goal here is video needs to be ubiquitous. It's a far richer form of communication than any other media. As a result, if I'm personally pushing video online, it's very difficult to justify using an environment that's only supported by 20 or even 80 per cent of users. You leave behind a significant portion of your user-base, at that point.

MM: Would you then suggest that Flash remains the unassailable incumbent, moving forward?

SN: At this point, for most content owners, yes. I think there are a few content owners that can use different environments. Take Netflix, for example - who's using SilverLight. Because their content and the user-experience around it are significantly compelling enough, they can essentially mandate that their users have to have SilverLight installed. Most users are willing to go through that process in order to watch a 2-hour video online, without having to wait for shipping.

But for the vast majority of content owners, Flash is still a pretty clear winner. We're watching SilverLight very carefully. I genuinely hope Microsoft pulls it off, well. I think it's a good market to have competition around. But today, Flash is the only ubiquitous player.
MM: In preparing for this interview, we'd discussed a basic model by which to characterize many of the distinctions that we'd like to draw out about online video. It was a simple $\mathrm{X}-\mathrm{Y}$ plot, where you've got quality as baseline, and on a vertical axis, you have functionality in terms of what you can do with video. Could you just walk us through this model?

SN: Sure.

Honestly, as we look through a lot of the top sites online and who's carrying a lot of their video, of course, YouTube has some of the broadest distribution. But YouTube has - primarily focused on less functionality and a lower-quality video with a broader distribution.

That said, they're making a great push to high-quality video. A lot of their new videos are transcoded at about 2 megabits per second. That's definitely driving them into a higher-quality video part of that plot.

At the same time, YouTube hasn't really pushed for a broader offering. Not something that's really compelling for the larger studios or higher-quality content owners. Those user-bases still primarily rely on YouTube for their user-base. They'll syndicate promotional content, there. But they'll keep their high-quality cards very close to their own sites, where they can monetize the content better and control more of that user-experience.

MM: Some of the features or properties of higher functionality would be tools for monetization, probably some metrics and/or content analytics, and probably some sort of control of the experience.

SN: Exactly. The basic ones that we break it up into are monetization, syndication, analytics, user-experience, branding - as well as the broader content management. How easy is it to simply manage that library that you control? MM: Yes.

SN: In general, we see that nobody has really tried to build out that entire suite - where you as a content owner can either have a one-stop shop or grab the necessary components that you need to integrate into our workflow.

The standard workflow a few years ago was that you'd download your own custom Flash player. You'd integrate it with DoubleClick's ad server. You'd grab Omniture and integrate it on the analytics side. And you'd do some of your 
own custom syndication, and work with your own CDNs to manage that CDN contract.

There were lots of moving parts that you had to manage and synchronize and coordinate.

MM: Right. You had to be really almost a systems integrator or a service integrator to be able to cobble all of that stuff together.

SN: Exactly.

We're seeing more and more people starting to group some of that together. But it really varies. Some people will integrate a third-party analytics solution. But how tightly integrated? That's sometimes the question.

MM: Let me just explore that one theme of content analytics, the one you described Omniture - is clearly an industry leader in terms of web analytics.

SN: Right.

MM: Most of their analytics reflect or derive from the content and metadata that's on the page, and not necessarily what's in the video.

There's very little content analytics in terms of the internals of the video stream. Yes? SN: For most offerings, yes. To give credit where credit's due, Visible Measures also has a good video analytics solution. They're the ones that partner with Brightcove.

A lot of our philosophy was - when we built it - we simply felt we could build it better. Leveraging the integration we could have with our own platform and our own player. Also, leveraging a lot of our own infrastructure experience.

We had really taken the route similar to Apple, 'I have my iPhone and it works incredibly well, and I have my MacBook Pro, which also works incredibly well'. But when I sync the two, and my iPhone syncs with my contact list - which is integrated into Mail - and it all works so seamlessly together, you get a sum that's greater than the individual parts.

We've really found that to be true with all of our individual components.

MM: With respect to content analytics, one of the primary reasons to understand who's doing what in terms of the video stream pretty much relates to monetization. For example, 'How can we wrap an ad around this?' Or insert an ad that's more specific to the context of the video, perhaps even the identity or preferences of the user.
Can you expand on that notion a little bit of how the analytics drive or close the loop, in terms of monetization?

SN: Yes - even indirectly. It's just simply knowing how much of your content users tend to watch. If they tend to always drop off after 2 minutes, or if they're skipping ahead, that changes your monetization strategy. If you have 30 per cent of your users abandon an industry ad, you should be looking for more intelligent places to place that ad.

You need to know, based on each individual part in your video stream, what that abandonment rate looks like for your user-base.

MM: That gets to the unspoken elephant in the room. That is, oftentimes, many of the people consuming online video are multitasking. Many times a video will play, but the consumer's not really engaged with it.

Do we have any other ways of measuring not just play or session time, but levels of engagement? Or are there distinct patterns of engagement represented in behavioral data?

SN: Yes. There definitely are. Especially as you track a user's behavior across videos.

When I finish a video, am I watching more videos with that time delay, as before I watch another video? Do I replay it? Am I seeking around and so on?

There's also the more-advanced topic and futuristic model, which I mentioned before the interactive video that we're exploring. There's an entirely new way of engaging with content, once you're able to unlock the individual elements within a video stream.

Same thing we're doing with a number of fashion companies right now - to monetize their runway shows and their other more marketing-focused material. If you actually enable a user to consume content in a leanforward consumption model as to the traditional lean-back consumption model, you get much better metrics. You get much better data about the user. And you're building a more compelling user-experience.

MM: What would be some facets or attributes of that lean-forward experience of consumption? SN: If I'm watching a sports game - getting to click on the athlete to pull up their stats. If I'm watching a fashion show, clicking on the model to learn more about their outfit. 
MM: This would entail, then, putting links inside the video stream.

SN: Essentially, yes.

MM: Maybe it's just an overlay or a hot zone?

SN: Essentially. I think a lot of the art and science are really married when you're trying to build a very scalable and robust technology that can make more advanced hotspots. Building on top of that with a very elegant user-experience that isn't necessarily just trying to sell somebody something, but getting them to engage more with that content.

The metrics are - of course - very clear. The more people are really engaging with their content, the longer they consume. The more monetization possibilities there are. Overall, it's a much better user-experience.

But it takes a lot of that art and science to actually enable that user-experience.

MM: Right.

We're going to fill out the rest of our little model in terms of quality and functionality.

SN: Yes.

MM: Where do you put a Brightcove or a Maven Networks in that framework?

SN: Maven, I'd put a little bit closer to mid- to low-end. Most of the models - as we honestly migrate their customers over to our platform and we look at the quality of content that they generally provide their customers - it's sort of a mid- to low-end. I believe it is encoded at roughly 500-600 kilobits per second.

As we look at a Brightcove, I think Brightcove is on the right track. They're definitely starting to push higher-quality video. Essentially, Brightcove today is leveraging the functionality opened up by Flash Media Server 3.5 and Flash 10 - which allow you to do adaptive rate delivery.

Today it works for about 60 per cent of users, but not 100 per cent of the user-base.

I think a global theme of what we're seeing is that adaptive bitrate is an absolute must. As we look at our global bandwidth metrics that we collect across seven or eight different CDNs on a daily basis, there is no clear bitrate that any user or any content owner can select, where they're delivering a great user-experience for all of their users.

The adaptive bitrate is an absolute core requirement. To our credit, we've actually been doing adaptive bitrate since early 2008. We just simply built it independent of Flash Media Server, and independent of Flash 10.

MM: How would you quickly summarize 'Adaptive Bitrate?'

$\mathbf{S N}$ : Adaptive bitrate is the ability to change the video quality for each individual user, mid-stream without interrupting the user-experience.

MM: That would both accommodate the bandwidth available to the individual user, and perhaps episodic demands on bandwidth that would obviously slow down the user's experience.

SN: Exactly.

We see adaptive bitrate moving toward ubiquity, as far as a peer. Everybody has to offer it. I think there are going to be different quality levels for adaptive bitrate.

There are going to be different deployments depending on whether they're using Flash or whether they're using SilverLight. Whether they're using a client-side technology or a service-side technology.

For the most part, in the next 2 years, it should be a standard offering across every provider.

MM: You've made a pretty good case for ubiquity, as being the basic criteria to satisfy, in terms of video services. Let's talk about all four screens, as you've talked about them before.

Can you just take us through how mobile devices and iPhones can participate in these video streams, vis-à-vis a service such as Ooyala. SN: For a lot of our content owners, it's really about not just pushing content to their site, but pushing content to wherever their users are able to consume it.

Take our average New Yorker or your average Brit. They spend a significant portion of their day traveling. Simply just commuting to and from work. If that's the case, there's a fantastic opportunity there to consume a lot of media content on their mobile device.

While it's very difficult that they'd read a long story on their iPhone, it's very easy to leverage the audio and video channels. To actually consume a much larger amount of information very quickly.

MM: What's the story, from your perspective, behind Apple's non-support of Flash? They say the quality is not there and they have to use Flash Lite, and so on. Is that the real story? Or is there something else going on? 
SN: I don't think it's ever, 'the real story'. I think there are lots of reasons that are probably going into it. Granted, I think there is some validity to the statements around Flash Lite not performing to the level that they require.

MM: And isn't their motivation basically to keep the iPhone more tightly linked to the Apple Store and iTunes?

SN: I wouldn't necessarily pass any judgment as to how much they're exercising that motivation. But it certainly exists.

MM: Clearly, over the near-term - anywhere from 1 year to 2 years - the iPhone will become a full digital citizen with respect to streaming high-quality video, I'd imagine.

SN: Yes. Honestly, to stream high-quality video to an iPhone is much easier. At that point, you're talking still sub-negative per second video.

That's far more tractable than trying to stream high-quality video to a desktop or even to the set-top box that powers your 50-inch plasma.

MM: Here I am with high-resolution video content and/or digital assets. I'm clearly broadcasting them out to my television networks' broadcasters. I'm making it available as a video on demand (VOD) for those people that are on cable or satellite TV.

I've clearly got it streaming to PCs around the world, and I'm kind of poking into mobile interest devices - be they Smart phones or other forms of Internet devices.

I might even want to push it into locationbased video. For example - at all the Wal-Mart stores or the JC Penney's and the video display presentation centers there. Right?

That was the case that The Platform made in terms of their business. They're now part of Comcast.

SN: Yes.

MM: Where do you put The Platform in this current matrix of ours? They seem to clearly understand the notion of not only having multiple codecs or playout devices, but then also wrapping content and commerce services around those.

SN: We really see The Platform as having had a broader span of integration points and functionality than almost anybody else in this space.

We generally see that people don't feel they're moving high enough up the stack. They're not really helping with more advanced monetization services. They're not helping with the higher-level user-experience functionality that they're really looking to do, especially if they focus on mobile and online.

We do bump into The Platform from time to time, but it really only is focused when you're hitting the very large media companies. We don't see any of the web portals. We don't see any of the tier two and tier three really ever using the Platform.

I think The Platform is focused on helping a very small subset of people fairly well. They haven't really expanded their functionality beyond that, to help a broader base.

I know they have a recent announcement about this with EdgeCast Networks. But still, we haven't actually seen it play out at all.

MM: As we've more or less filled out - or have we really talked about - the top righthand corner of that? In terms of high quality and high functionality and in terms of who really occupies that space? Or is that yet occupied?

SN: In all honesty, no, I don't think it's yet occupied. I think there are a number of us vying for it. I think the problem probably is that a number of other players have clearly staked out their space.

Move Networks is now trying to vie for the high-functionality, high-quality experience space. They're staying very focused on what they do, and doing it very well.

We're obviously vying for that. We think it's a very big industry. We're trying to help make that pie bigger for everybody. In the course of really building out a platform that provides all the necessary components for our content owners to build a successful business online, we are expanding a lot of that functionality.

We generally believe that we're the frontrunners, but there's no clear winner in that space - by any stretch of the imagination. MM: Usually in an open field currently not served by anyone, a catalytic event occurs that then creates a huge vacuum of demand that ultimately forces or induces a company to emerge as a winner.

Events - catalytic or otherwise - would really cause that high-functionality and high-quality video space to really coalesce into a real market. SN: Absolutely. The Dow being close to 7900 . As we're really seeing it - and speaking to the evolution of Ooyala's platform, here - we're 
seeing an extremely high demand for our platform in the current market. Especially the modularity components of our platform, from the absolute top-tier providers. They're forced to outsource a lot of their efforts to really reduce their overall spend.

The name of the game for a lot of these divisions in these individual companies is simply to survive 2009. They can't do that when they're spending millions of dollars on staffing and millions of dollars on technology, to do something that we Ooyala can simply provide for them at a quarter of the cost or a tenth of the cost of what they're currently paying.

We have an entire team focused on all of that platform and on all of that technology. They're simply just outpacing any efforts that they can actually rally internally.

The current market is forcing people to make intelligent decisions with their money. We're going to see a lot of these smaller players in this space drop out in 2009 and 2010. But we're going to see those who can really push their platform into that top right corner, there.

We're going to continue to see people get more and more traction from a lot of their sales channels. There will be a very clear winner, I think, in the next 2 years.

MM: You said that basically, greater functionally consists of monetization, analytics, user-experience, branding, content-management and so on.

In particular, I'd like you to speak to the area of metadata - and more specifically, the orchestration or integration of three sets of metadata.

To set that up, many advertisers - brand marketers - seek the holy grail of automated spot-insertion into a known demographic or psychographic profile.

SN: Yes.

MM: In order to make this happen, you have to really have a pretty good idea in terms of the user-profile, and a really good idea in terms of the content in which it's showing up. As well, a really good set of metadata on terms of the ad, itself. In terms of target markets and so on.

In terms of correlating these three sets: the user-profile, the context and the actual ads, can you take us through how you see that evolving? And to what extent automated ad insertions will become reality?
SN: I genuinely believe they will. I think there's a very limited set of companies that can make it a reality.

I think as we're finding even today in the ad space, there are lots of poor-man's implementations. They get maybe 60 per cent of the benefit advanced strategies like that.

But to really do it properly, it takes a lot of technological innovation. There are very few companies that can really pull that off. Google, of course, is one of them. We're predicting that Ooyala will be another.

But as we really look at what it takes - a huge amount of both computing horsepower, as well as simple elegant solutions that you can architect - really, you're trying to marry a massive amount of data with another core requirement. That is, you need a massive user-base.

You can't get dynamic insertion of ad spots if you're only getting 10 views on a piece of content. There's just simply not enough data to really learn about it, there.

So we really see it as a spectrum.

If you tell me every possible components of a video, and tell me incredibly accurately - I don't need a whole lot of impressions to do dynamic experimentation on where that ad should sit. But that's not really tractable.

No computer vision algorithm. No audio extraction algorithm. And no human's really going to be able to accomplish that for you.

You really need just sheer volume. The experiments we ran back at Google to really optimize a lot of the advertising user-experience there required millions or tens of millions or hundreds of millions of data points to get a tractable data set, to really extract maximum value for users and advertisers.

I think those are the core components, there. And that's why there's such a limited set of technology providers that could actually achieve this.

You need sheer volume. You need a lot of users viewing a lot of content and a lot of advertising going in. And you need the technological acumen to make it a reality.

Does that answer your question? MM: It sure does.

That leads us then to the logical next-step or question; as advertisers seek to optimize their media mix, and as Google has really kind of disrupted the whole media-mix optimization 
with ad words and other forms of fact-based media placements. In terms of exactly 'What am I getting for my ad spend?'

Can you really address the evolution of how media buys will emerge from a planning and/or media-mix optimization, with the services that you're talking about becoming more ubiquitous? SN: I think it's going to be become a much more data-driven process. The fundamental expectation online is that, regardless of what type of medium you're delivering your ads through, whether it's banner ads or companions or in-streams or an interactive-based model, advertisers are going to spend an experimental budget once or twice, to see how things work. But beyond that, offline media has yet to become that successful model that everybody's comfortable with.

If you're trying to displace that with online advertising, you have to come to the table with better metrics.

MM: That was always the strength of online whether it was a click-through or other form of behavioral data, online always had the advantage of saying, 'Here's a known devil versus an unknown devil'.

SN: Right.

But I think at the same time, that's been for online, page-based - but not necessarily online video.

MM: Right.

SN: I don't think we've hit the point of where we have a comparable level of granularity, and a very different medium to really keep pushing a lot of advertising dollars. Especially in 2009, where budgets are shrinking, there will be a required amount of accountability - so people can see their return on investment (ROI).

MM: That's also part of your story, in terms of the catalytic event. As companies continue to hunker down and get much more practical about their marketing spend, that will accelerate their gravitating toward more and more transparent and accountable ways of spending. SN: Absolutely. Yes.

If companies want to play in this space, they're going to have to cater to the analytics-driven crowd.

MM: Yes.

As we step forward and start to summarize our conversation here today, perhaps you can take a fast-forward and paint a picture of a future proof, in terms of the structure of the market 5 years from now, and some of the roadmaps by which Ooyala's getting there.

SN: I think we're going to be delivering a lot more content over internet protocol (IP). Naturally. That's really going to enable much richer functionality on set-top boxes.

MM: Before you move on from that, who do you think is going to be the winner? Either in terms of service and/or device, for IPTV?

SN: Honestly, I love TiVo. I have a TiVo box. I have a VooDoo box. I pretty much have all the toys. But I don't see them winning. They don't have the footprint.

Months after Comcast decided they wanted to get into the digital video recorder (DDR) space and really deploy their strategy; they were the largest and most-popular DDR on the market.

I see companies like Motorola - who manufacturers the Comcast DDR, I see the cable providers really being in the best position to do that. They're very concerned about protecting their domain, as far as delivering contention to the TV. They're incredibly incentivized to protect that revenue stream.

MM: Yes. So it'll be probably some device attached or tightly coupled with the cable and satellite broadcast networks.

SN: I think it's likely.

The other one I think is potentially an even more-likely candidate would be some of the TV manufacturers. I think Sony's always been an innovative corporation. If I'm able to build a TV that connects directly over IP, do I need Comcast any more?

MM: No. You don't. You just need to have a stable high-speed web connection. Right? SN: Exactly.

They're the other big players that I think have enough financial backing to make that a reality. It's a very expensive game.

TiVo has struggled, because it's so difficult to build a box for $\$ 200$ or 300 that they can make any reasonable profit off of, and still actually capture a broad-enough user-base.

MM: This kind of reminds me of the early 1980s, in terms of to what extent the PC first came into existence. Client-server computing started to emerge, and so on.

Is the device essentially going to be a dumb terminal, or is it going to be a fully functioning 
computer that occasionally needs to go online to do stuff?

SN: Right.

We definitely see the trend. They are moving toward smart devices - as every manufacturer really looks to expand their business and actually capture more revenue.

If you look at CES this year, TVs are getting thinner. But they can't really get that much bigger. All of the manufacturers are really looking at other places, where they can innovate, to really compel their customers to purchase new models. MM: Wouldn't that make the case then that the TV manufacturers basically come up with some computing platform?

Clearly, that Smart Device needs an operating system (OS). More importantly, it needs to have a developer ecosystem supporting it with all kinds of continuous upgrades.

That's where the TiVo's and so on have struggled mightily. They haven't really had a developer ecosystem expanding the function off their income statement.

SN: Yes. Well, TiVo actual does. It's just an incredibly neglected ecosystem. When we were working with it in the early days, it was just too difficult to ever deploy anything on.

But yes, Sony, Toshiba and a number of other manufacturers are putting time into building these native OS-based environments on their TVs. They're functioning a lot more like PCs with a really big screen.

MM: It seems to me that there are other areas of the world that have much more capacious bandwidth to the home than we do here in the United States.

It seems that perhaps even in a third world or developing country, there's heavy incentive to bring broadband to the home for economic reasons.

It seems to me that there would be an open source TV platform that might take off like wildfire. All of a sudden you'd have several tens - maybe even hundreds of - millions of devices there. Outside of, for example, the United States or the EU. They would ultimately force or create the scale of manufacturing to really bring a low-cost, fully featured computing-device TV to a consumer market. SN: Yes. You definitely bring up a good point. In the US, bandwidth to the home is not so sufficiently high that it's compelling.
Cable-based offerings are robust enough that the cost of switching is significantly high.

If you look at Japan, the bandwidth to the high is at an extremely high capacity. The context to switch is potentially less. Especially if there's no viable alternative.

I think the challenge will always be, 'Do I have to buy my TV and another device that goes with it?' Or, 'Can I get a device mixed in with my TV?' The TV manufacturers would, of course, love that.

How that really plays out ... Are they going to be proprietary-based systems? Probably in the short term, they will be. That's the usual pattern that people don't tend to learn from. And how long does it take until there's a more open and collective system that everybody can build and deploy to?

MM: Another possibility of disruption in terms of that high-quality, high-functionality space is essentially an iPhone from Apple. It's already got a full UNIX kernel in there. It's got a really robust developer ecosystem. And if it had a faster microprocessor, it could clearly drive a large-screen TV or a larger microprocessor with a video RAM and a video processor. Right? SN: Right.

MM: Is it conceivable that the disruption comes not from traditional device manufacturers, but from a mobile device on steroids?

SN: I think that's genuinely conceivable, but I think we're multiple years away. On the hardware side, to get that small of a handheld device - to be able to power even SDTV, let along high defination (HD) - but it is conceivable.

I think today, we're still looking for true HD support in the browser. That's difficult. That will be cracked, first. Then of course the set-top boxes and standard PCs - to power HD on TV.

But I would not be surprised if at some point in the coming years it is enabled by a mobile device.

MM: As we start to wrap this up, are there any last comments you'd like to summarize with? SN: No. I think we've had a good discussion here. As we generally look at it, I love looking at the futuristic stuff. We definitely see our role as very clearly to provide the most successful platform across the broadest user-base. But to also help accelerate that evolution toward these more-advanced and futuristic deployments. 
I think the buzzword is that as a small company it's foolish to drop into this large of an industry and think you can revolutionize it - but you can absolutely accelerate that rate of evolution.

That's really been our continual goal. MM: Right.

In fact, isn't that the underlying innovation and philosophy of Google, in terms of small projects and small teams - rapidly executed across multiple parallel paths - that ultimately add up to significant transformations or disruptions? But in and of themselves, are humble and small-scale? SN: Exactly.

MM: Sean - Thank you so much for the interview.

SN: Likewise. I definitely appreciate the time. 